\title{
Circularly polarized waves in a plasma with vacuum polarization effects
}

\author{
J. Lundin, L. Stenflo, G. Brodin, M. Marklund and P. K. Shukla \\ Department of Physics, Umeå University, SE-901 87 Umeå, Sweden
}

August 24, 2021

\begin{abstract}
The theory for large amplitude circularly polarized waves propagating along an external magnetic field is extended in order to include also vacuum polarization effects. A general dispersion relation, which unites previous results, is derived.
\end{abstract}

Circularly polarized waves can be exact solutions of the fluid and Maxwell equations (e.g. Refs. $[1,2])$. Thus, it is possible to study large amplitude waves propagating along an external magnetic field in a plasma, taking also relativistic effects into account. Here we shall demonstrate that it is comparatively easy to include quantum electrodynamical field (QED) effects [3,4] in such an analysis, also for the general case with a multicomponent plasma.

Let us start with the equations of continuity and momentum for each species (denoted by index $s$ ) in a cold, multi-component, relativistic, magnetized plasma

$$
\partial_{t} N_{s}+\nabla \cdot\left(N_{s} \mathbf{v}_{s}\right)=0
$$

and

$$
\partial_{t} \mathbf{p}_{s}+\mathbf{v}_{s} \cdot \nabla \mathbf{p}_{s}=q_{s}\left(\mathbf{E}+\mathbf{v}_{s} \times \mathbf{B}\right) .
$$

Here $N$ is the particle density, $\mathbf{v}$ the velocity, $q$ the charge, $m$ the rest mass, $\mathbf{p}=m \mathbf{v} /(1-$ $\left.v^{2} / c^{2}\right)^{1 / 2}$ the momentum and $c$ the speed of light in vacuum. Furthermore, we use the Maxwell's equations for the electric and magnetic fields $\mathbf{E}$ and $\mathbf{B}$, i.e.

$$
\nabla \times \mathbf{E}=-\partial_{t} \mathbf{B}
$$

and

$$
\nabla \times \mathbf{B}-c^{-2} \partial_{t} \mathbf{E}=\mu_{0} \sum_{s} N_{s} q_{s} \mathbf{v}_{s}+\mu_{0} \mathbf{J}_{\mathrm{vac}},
$$

where the vacuum current $\mathbf{J}_{\mathrm{vac}}$ (after having corrected an obvious misprint in the definition of the coupling constant in Ref. [3]) is given by (e.g. Refs. [5,6])

$$
\begin{aligned}
\mathbf{J}_{\mathrm{vac}}= & -2 \kappa \varepsilon_{0}^{2}\left\{c^{2} \nabla \times\left[2\left(E^{2}-c^{2} B^{2}\right) \mathbf{B}-7(\mathbf{E} \cdot \mathbf{B}) \mathbf{E}\right]\right. \\
& \left.-\partial_{t}\left[2\left(E^{2}-c^{2} B^{2}\right) \mathbf{E}+7 c^{2}(\mathbf{E} \cdot \mathbf{B}) \mathbf{B}\right]\right\},
\end{aligned}
$$

where $\kappa=2 \alpha^{2} \hbar^{3} / 45 m_{e}^{4} c^{5}$ is the nonlinear coupling constant and $\alpha=e^{2} / 4 \pi \hbar c \varepsilon_{0}$ is the fine structure constant. Here $e\left(=-q_{e}\right)$ is the elementary charge, $m_{e}$ the electron rest mass, 
$\varepsilon_{0}$ the vacuum permittivity, $\mu_{0}$ the vacuum permeability and $\hbar$ is $2 \pi$ times the Planck constant. The expression (5) for the vacuum current is valid for field strengths lower than the critical field strength, $E_{\text {crit }}=m_{e}^{2} c^{3} / \hbar e$, and for frequencies lower that the Compton frequency, $\omega_{e}=m_{e} c^{2} / \hbar$. These equations have been recently used by Di Piazza et al. [6].

We now consider the propagation of a circularly polarized wave along an external constant magnetic field $B_{0 z} \hat{\mathbf{z}}$. Following Ref. [2] we first note that $E^{2}$ as well as $B^{2}$ are then constants and that $\mathbf{E} \cdot \mathbf{B}=0$. This means that

$$
\mathbf{J}_{\mathrm{vac}}=-4 \kappa \varepsilon_{0}^{2}\left(E^{2}-c^{2} B^{2}\right)\left(c^{2} \nabla \times \mathbf{B}-\partial_{t} \mathbf{E}\right) .
$$

Inserting Eq. (6) into Eq. (4) we thus find our new equation

$$
\nabla \times \mathbf{B}-c^{-2} \partial_{t} \mathbf{E}=\mu_{0} \mathbf{J}_{\mathrm{eff}},
$$

where

$$
\mathbf{J}_{\text {eff }}=\sum_{s} N_{s} q_{s} \mathbf{v}_{s}\left\{1-\frac{2 \alpha}{45 \pi}\left[\left(n^{2}-1\right)\left(\frac{E_{0 \perp}}{E_{\text {crit }}}\right)^{2}+\left(\frac{c B_{0 z}}{E_{\text {crit }}}\right)^{2}\right]\right\}^{-1} .
$$

Here $n=k c / \omega$ is the index of refraction, and $E_{0 \perp}$ is the electric field amplitude of our right hand circularly polarized wave $\mathbf{E}=E_{0 \perp}[\cos (\omega t-k z) \hat{\mathbf{x}}+\sin (\omega t-k z) \hat{\mathbf{y}}]$. The rest of the calculations are now straightforward. Introducing the symbols $E_{ \pm}=E_{x} \pm i E_{y}$, $B_{ \pm}=B_{x} \pm i B_{y}$ and $v_{ \pm}=v_{x} \pm i v_{y}$ we thus adopt the analysis of Ref. [2], noting that

$$
\begin{gathered}
E_{ \pm}=E_{0 \perp} \exp ( \pm i \omega t \mp i k z), \\
B_{ \pm}= \pm i \frac{k}{\omega} E_{ \pm}
\end{gathered}
$$

and

$$
v_{ \pm s}=\mp \frac{i q_{s}}{\gamma_{s} m_{s}\left(\omega+\omega_{c s}\right)} E_{ \pm}
$$

where $\omega_{c s}=q_{s} B_{0 z} / \gamma_{s} m_{s}$ is the relativistic gyrofrequency of particle species $s$ and $\gamma=$ $\left(1-v_{0}^{2} / c^{2}\right)^{-1 / 2}$ is the Lorentz factor which in this case is a constant with $v_{0}^{2}=v_{+} v_{-}$. Usually, we define the relativistic plasma frequency as $\omega_{p s}=\left(q_{s}^{2} N_{0 s} / \gamma_{s} m_{s} \varepsilon_{0}\right)^{1 / 2}$. Here, however, instead of $\omega_{p s}$ we will in the dispersion relation find the effective plasma frequency

$$
\Omega_{p s}=\omega_{p s}\left\{1-\frac{2 \alpha}{45 \pi}\left[\left(n^{2}-1\right)\left(\frac{E_{0 \perp}}{E_{\text {crit }}}\right)^{2}+\left(\frac{c B_{0 z}}{E_{\text {crit }}}\right)^{2}\right]\right\}^{-1 / 2} .
$$

By combining Eqs. (7) and (8) we obtain the dispersion relation

$$
n^{2}-1=-\sum_{s} \frac{\omega_{p s}^{2}}{\omega\left(\omega+\omega_{c s}\right)}+\frac{2 \alpha}{45 \pi}\left[\left(n^{2}-1\right)\left(\frac{E_{0 \perp}}{E_{\text {crit }}}\right)^{2}+\left(\frac{c B_{0 z}}{E_{\text {crit }}}\right)^{2}\right]\left(n^{2}-1\right) .
$$

The theory for a left hand circularly polarized wave is quite similar and leads to the same dispersion relation but with $\omega_{c s} \rightarrow-\omega_{c s}$. 
We note that Eq. (9) agrees with Eq. (13) in Ref. [7] in the special case of an ultrarelativistic electron-positron plasma, and with Eq. (9) in Ref. [8] for a dusty plasma (if we correct for a misprint in Refs. [7] and [8]; $4 \alpha$ should be $2 \alpha$ ). Eq. (9) is exact within the basic model we have used in the present paper.

As $\alpha E_{0 \perp}^{2} / E_{\text {crit }}^{2}$ in general is a very small parameter, we can next expand the solution of Eq. (9) to obtain

$$
\begin{array}{r}
n^{2}=1-\sum_{s} \frac{\omega_{p s}^{2}}{\omega\left(\omega+\omega_{c s}\right)}-\frac{2 \alpha}{45 \pi}\left(\frac{c B_{0 z}}{E_{\text {crit }}}\right)^{2} \sum_{s} \frac{\omega_{p s}^{2}}{\omega\left(\omega+\omega_{c s}\right)} \\
+\frac{2 \alpha}{45 \pi}\left(\frac{E_{0 \perp}}{E_{\text {crit }}}\right)^{2}\left[\sum_{s} \frac{\omega_{p s}^{2}}{\omega\left(\omega+\omega_{c s}\right)}\right]^{2},
\end{array}
$$

which agrees with the result of Ref. [6] in the limit $B_{0 z}=0$.

New modes in unmagnetized QED plasmas have been reported previously [9]. The calculation were then extended to magnetized QED plasmas with ultrarelativistic ions. Such plasmas can support amplitude-dependent electromagnetic ion waves [10]

$$
\omega \approx \frac{k^{2} c^{2}}{\omega_{p i}^{2}} \omega_{E i},
$$

where $\omega_{p i}$ is the ion plasma frequency and $\omega_{E i}=e E_{0 \perp} / \mathrm{cm}_{i}$, as well as new modes in a dust-ion plasma [11], and QED effects can then play a key role [8,11]. Di Piazza et al. demonstrated in Ref. [6] that the QED effects can be important in wave propagation studies, even for the case where $B_{0 z}=0$. They showed that the last term in Eq. (10) can be larger than the sum of the first two terms on the right hand side of Eq. (10) near the plasma cut-off frequency. Thus, they demonstrated enhancement of the vacuum polarization effects when the frequency is close to $\omega_{p}$. Our dispersion relation shows that there is no analogous enhancement of the QED contribution from the external magnetic field near the plasma cut-off frequency. This is because the 2 nd and the $3 \mathrm{rd}$ term on the right hand side of Eq. (10) have the same sign. Thus, the QED contribution from the external magnetic field will always remain smaller than the classical contribution in Eq. (10) unless $c B_{0 z} \gg E_{\text {crit }}$, but this condition lies outside the validity of our model. The QED contribution from the external magnetic field may still be larger than that of the last term in Eq. (10), however, in particular if $c B_{0 z}>E_{0 \perp}, \omega_{p}<\omega$ and if $\left(\omega+\omega_{c s}\right)$ is not close to resonance. But in this regime there is no enhancement of the vacuum polarization effects as described in Ref. [6].

It is also interesting to note that the effective plasma frequency $\Omega_{p}$ is different from $\omega_{p}$. For example if $k \approx 0$ we have

$$
\frac{\Omega_{p s}}{\omega_{p s}}=\left[1+\frac{2 \alpha}{45 \pi E_{\text {crit }}^{2}}\left(E_{0 \perp}^{2}-c^{2} B_{0 z}^{2}\right)\right]^{-1 / 2} .
$$

However, for field strengths available in laboratory experiments, this ratio is close to unity.

Another example of interest concerns the free electron laser (FEL) [12-14]. Considering magnetostatic wigglers, i.e. letting $\omega \rightarrow 0$, in a one-component electron plasma with drift velocity $v_{z e}$ and gyrofrequency $\omega_{c e}$, the dispersion relation (9), using the laboratory frame rather than the electron rest frame, becomes

$$
k^{2} c^{2}\left[1-\frac{2 \alpha}{45 \pi}\left(\frac{c B_{\mathrm{tot}}}{E_{\mathrm{crit}}}\right)^{2}\right]=\frac{\omega_{p e}^{2} k v_{z e}}{\omega_{c e}-k v_{z e}}
$$


where we have introduced the total magnetic field $B_{\text {tot }}=\sqrt{B_{0 \perp}^{2}+B_{0 z}^{2}}$ and the magnetic field amplitude $B_{0 \perp}$ of the wiggler field. For $c B_{\text {tot }} \ll E_{\text {crit }}$, Eq. (11) agrees for example with (8) in Ref. [14]. Thus our formula (11) generalizes the FEL dispersion relation to account for field strengths approaching the Schwinger critical field. While current laboratory applications have a long way to go in order to have fields in this range, there nevertheless exist schemes attempting to reach such regimes [15]. We note that the particle orbits in FELs are nontrivial $[13,14]$ and that the helical equilibria may not be stable [13]. In the example above we have also supposed the presence of a stationary background assuring neutrality, which means that there are several limiting assumptions concerning forthcoming experiments [6]. Thus we hope that the readers will find interest in further extending the basic theory of Ref. [2].

In the present manuscript we have derived the dispersion relation for large amplitude circularly polarized electromagnetic waves propagating parallel to an external magnetic field in a multicomponent plasma. Our dispersion relation (9) accounts for vacuum polarization and magnetization (both due to the external field and the wave field), fully relativistic quiver velocities and general drift velocities of the plasma species, and applies for arbitrary ratios of the characteristic frequencies of the problem. As a consequence, such different waves as Alfvén modes, whistler modes and large amplitude laser modes are covered by the general theory. Considering specific regimes, Eq. (9) reduces to several special cases found in the literature. Besides uniting the results of many previous works in a single formalism, the results presented here can find applications to wave propagation in astrophysical contexts, e.g. in magnetar atmospheres [16], as well as in the next generation of FELs [15].

We consider the present work as a necessary prerequisite to a more complete theory which also includes a full stability analysis [2]. Thus it has for example been shown [1] that a single cold electron beam in an unmagnetized plasma is unstable if $k=0$ and if

$$
\left(1+\frac{\omega_{p e}^{2}}{K^{2} c^{2}}\right)^{-1}<\frac{v_{0}^{2}}{c^{2}+v_{0}^{2}}<\frac{K^{2} c^{2}}{\Omega^{2}}
$$

where $\Omega$ and $K$ are the frequency and wavenumber of the perturbations. The vacuum current will however modify all stability criteria.

\section{References}

[1] N.L. Tsintsadze and L. Stenflo, Phys. Lett. A 48, 399 (1974).

[2] L. Stenflo, Phys. Scr. 14, 320 (1976).

[3] G. Brodin, M. Marklund and L. Stenflo, Phys. Rev. Lett. 87, 171801 (2001). Here, 20 in the definition of $\xi$ should be replaced by 2 .

[4] M. Marklund and P. K. Shukla, Rev. Mod. Phys. 78, 591 (2006).

[5] M. Soljačić and M. Segev, Phys. Rev. A 62, 043817(R) (2000).

[6] A. Di Piazza, K. Z. Hatsagortsyan and C. H. Keitel, Phys. Plasmas 14, 032102 (2007). 
[7] M. Marklund, P.K. Shukla, L. Stenflo, G. Brodin and M. Servin, Plasma Phys. Control. Fusion 47, L25 (2005).

[8] M. Marklund, L. Stenflo, P.K. Shukla and G. Brodin, Phys. Plasmas 12, 072111 (2005).

[9] L. Stenflo, G. Brodin, M. Marklund and P.K. Shukla, J. Plasma Phys. 71, 709 (2005).

[10] L. Stenflo and N.L. Tsintsadze, Astrophys. Space Sci. 64, 513 (1979).

[11] L. Stenflo and P.K. Shukla, IEEE Trans. Plasma Sci. 29, 208 (2001).

[12] T. Kwan, J. M. Dawson and A. T. Lin, Phys. Fluids 20, 581 (1977).

[13] A. Goldring and L. Friedland, Phys. Rev. A 32, 2879 (1985).

[14] H. P. Freund, R. A. Kehs and V. L. Granatstein, Phys. Rev. A 34, 2007 (1986).

[15] A. Ringwald, Phys. Lett. B 510, 107 (2001).

[16] M. G. Baring and A. K. Harding, Astrophys. J. 507, L55 (1998). 